\title{
Rapid Mechanochemical Synthesis of Amides with Uronium-Based Coupling Reagents, a Method for Hexa-amidation of Biotin[6]uril
}

Tatsiana Dalidovich, Kamini A. Mishra, Tatsiana Shalima, Marina Kudrjašova, Dzmitry G. Kananovich* and Riina Aav*

Tallinn University of Technology, School of Science, Department of Chemistry and Biotechnology, Akadeemia tee 15, 12618 Tallinn, Estonia

\begin{abstract}
Solid-state reactions using mechanochemical activation have emerged as solventfree atom-efficient strategies for sustainable chemistry. Herein we report a new mechanochemical approach for the amide coupling of carboxylic acids and amines, mediated by combination of (1cyano-2-ethoxy-2-oxoethylidenaminooxy)dimethylaminomorpholinocarbenium hexafluorophosphate (COMU) or $\quad N, N, N^{\prime}, N^{\prime}$-tetramethylchloroformamidinium hexafluorophosphate (TCFH) and $\mathrm{K}_{2} \mathrm{HPO}_{4}$. The method delivers a range of amides in high 70$96 \%$ yields and fast reaction rates. The reaction protocol is mild, maintains the integrity of the adjacent to carbonyl stereocenters, and streamlines isolation procedure for solid amide products. Minimal waste is generated due to the absence of bulk solvent. We show that $\mathrm{K}_{2} \mathrm{HPO}_{4}$ plays a dual role, acting as a base and a precursor of reactive acyl phosphate species. Amide bonds from hindered carboxylic acids and low-nucleophilic amines can be assembled within 90 min by using TCFH in combination with $\mathrm{K}_{2} \mathrm{HPO}_{4}$ or $\mathrm{N}$-methylimidazole. The developed mechanochemical liquid-assisted amidation protocols were successfully applied to the challenging couplings of all six carboxylate functions of biotin[6] uril macrocycle with phenylalanine methyl ester, resulting in an $80 \%$ yield of highly pure hexa-amide-biotin[6] uril. In addition, fast and high-yielding synthesis of peptides and versatile amide compounds can be performed in a safe and environmentally benign manner, as verified by green metrics.
\end{abstract}

Keywords: mechanochemistry, solvent-free chemistry, amides, peptides, amide coupling reagents, macrocycle, hemicucurbituril, green metrics

\section{INTRODUCTION}

Amide bond is widespread in both natural compounds and artificial materials. It occurs in molecules fundamental to life, such as peptides and proteins, as well as in synthetic polymers and in a massive array of pharmaceuticals. In fact, amide preparation from carboxylic acids and amines 
represents the most frequently applied chemical transformation in drug production and comprises about $25 \%$ of the current medicinal chemistry synthetic toolbox. ${ }^{1}$ As a consequence of its wide usage, the development of sustainable amidation methods was listed among the top green chemistry research priorities by the American Chemical Society Green Chemistry Pharmaceutical Roundtable (ACS GCIPR) in $2007^{2}$ and has been retained in the recent revision. ${ }^{3}$ Although direct condensation of carboxylic acids and amines with water as a single by-product can be considered a "green" landmark in the field, it remains impractical because of the process's harsh reaction conditions $\left(\mathrm{T}>100{ }^{\circ} \mathrm{C}\right.$ ) and limited substrate scope..$^{4-6}$ A robust method of amide synthesis commonly requires prior activation of a carboxylic function to replace $\mathrm{OH}$ with a better leaving group. ${ }^{7-9}$ Notably, this is also the case in biosynthetic pathways, including the translation process and non-ribosomal enzymatic transformations. ${ }^{10-13}$ For laboratory and industrial use, vast numbers of amide coupling reagents, performing in situ activation of carboxylic acid, have been developed in the quest for faster, milder, and high-yielding amidation protocols. ${ }^{14,15}$ Low atom economy of these reagents and accompanying safety issues are their major drawbacks, which has incited the development of alternative approaches. ${ }^{16-19}$ Important advancements have thus far followed traditional solution-based approaches; however, solvent is actually responsible for $80-90 \%$ of mass consumption in a typical chemical process and also plays a major role in overall toxicity. ${ }^{20}$ In this way, solvent greatly outperforms the contributions of reagents themselves. Hazardous solvents like DMF and DCM are preferred in industrial amide synthesis, reinforcing both environmental and safety concerns. ${ }^{17,21}$ Therefore, the application of solvent-free techniques represents an efficient way to improve the overall process mass intensity and to prevent generation of hazardous waste. Recent advances in mechanochemistry and its related fields have established solvent-free reactions as environmentally friendly tools to perform chemical transformations that are no less efficient than the conventional solution-based chemistry. ${ }^{22-24}$

In the area of amide synthesis, the benefits of solvent-free techniques have not remained unnoticed and have been previously demonstrated in numerous studies (Scheme 1). ${ }^{25-27}$ For example, mechanosynthesis of various amides and peptides has been performed from a series of activated carboxylic acid derivatives, such as $N$-carboxyanhydrides $;{ }^{28,29} \mathrm{~N}$-hydroxysuccinimide esters; ${ }^{30} \mathrm{~N}$ acyl benzotriazoles. ${ }^{31} \mathrm{~N}$-Acyl imidazoles ${ }^{32}$ and acyloxytriazine esters ${ }^{33}$ have been produced mechanochemically from carboxylic acids prior to reacting with amines. Notably, even papain enzyme can catalyze the formation of peptides from the corresponding amino acid building blocks 
under solvent-free conditions. ${ }^{34,35}$ In addition, direct coupling of amines with carboxylic acid has been demonstrated by using 1-ethyl-3-(3-dimethylaminopropyl)carbodiimide (EDC) as a coupling reagent. ${ }^{36,37}$ In general, EDC-mediated transformations have shown remarkably short reaction times (typically within 10-30 min), high yields, and simple work-up protocols.

Scheme 1. Overview of the State-of-Art Mechanochemical Amidation Approaches and Outline of the Current Work

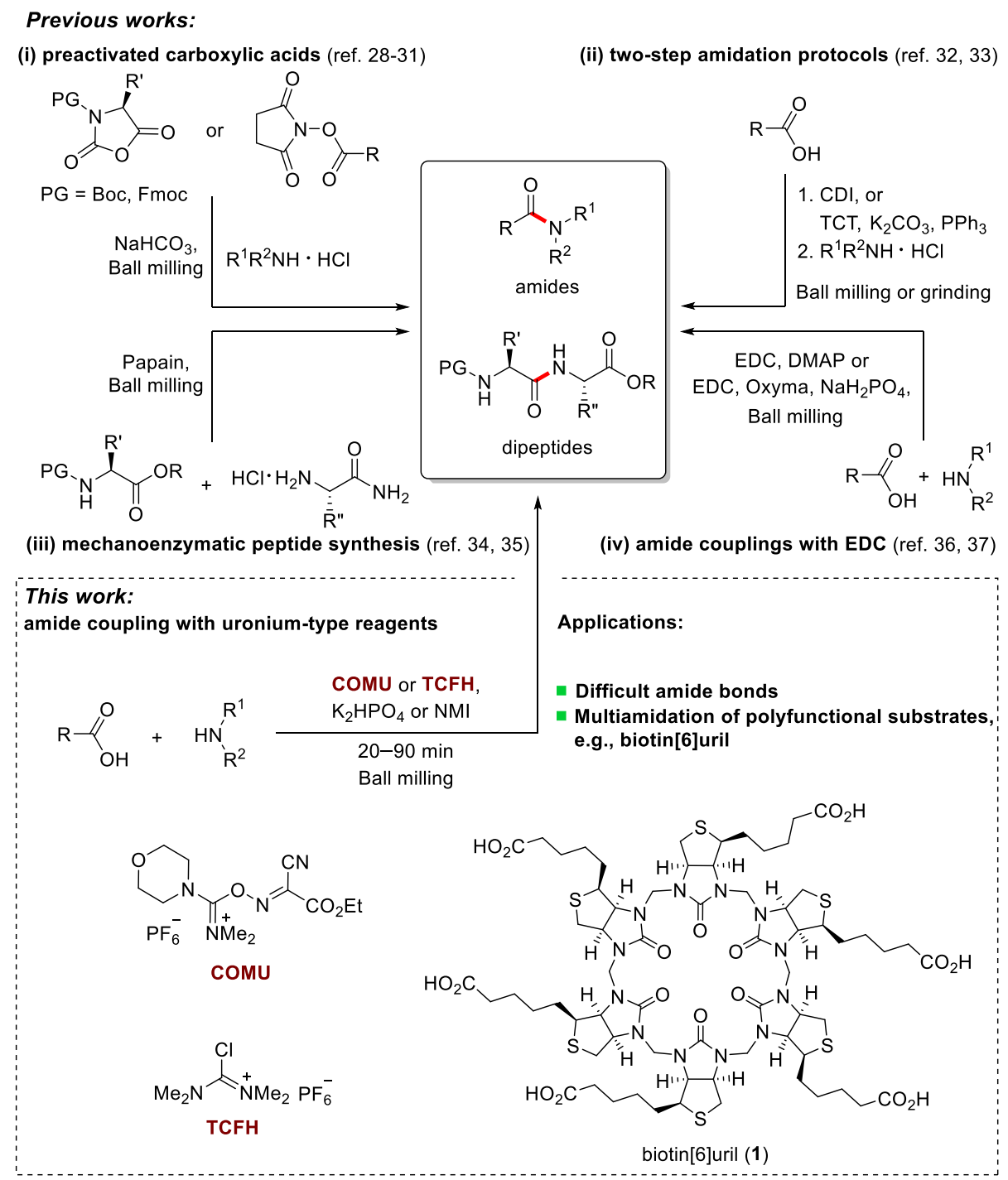

Following these prominent earlier contributions, ${ }^{24-33}$ we aimed to further expand the scope and synthetic utility of the mechanochemical amidation methods. The current research was impelled by three objectives: First, most of the amide coupling reagents are simply not efficient enough for a range of substrates, ${ }^{8}$ which require expansion of the established one-step mechanochemical 
amidation protocols beyond the previously applied EDC; for that purpose, in this work we mapped the coupling efficiency of uronium-type reagents (COMU and TCFH, Scheme 1) on several carboxylic acid/amine pairs. Second, the scope of previously published mechanochemical approaches was evaluated based mainly on peptide synthesis, while the challenging couplings of sterically hindered carboxylic acids and low-nucleophilic amines remained virtually unproven; here we demonstrated that such difficult amide bonds can also be assembled under solvent-free conditions. Implementation of the two objectives mentioned above was required as a prerequisite for the third objective as our ultimate goal. Due to the interest of our group in the synthesis and supramolecular applications of macrocyclic host molecules, ${ }^{38-43}$ we required a robust procedure for amide-functionalization of biotin[6]uril macrocycle (1), ${ }^{44-46}$ to access the family of modified biotin[6]uril hosts. Despite the apparent ease of such a transformation, it also presented a substantial challenge: six-fold stepwise amidation of carboxylate groups in $\mathbf{1}$ is inevitably accompanied by accumulation of the "failed" underfunctionalized products if incomplete coupling occurs at each step. Limited solubility of $\mathbf{1}$ in the common organic solvents dictates additional practical inconvenience of the traditional solution chemistry; in fact, only dipolar aprotic solvents like DMF can be used. Here we showed that application of solvent-free techniques, additionally reinforced with the reactive uronium-type amide coupling reagents, allows the desired functionalization of $\mathbf{1}$ in a high-yielding, scalable, and sustainable manner, avoiding harmful solvents or significant reagent excess.

\section{RESULTS AND DISCUSSION}

Development of Mechanochemical Amidations with Uronium-Type Reagents. At the outset, amide coupling of Cbz-protected L-phenyl alanine (2) and ethyl 4-aminobenzoate (benzocaine, 3), mediated by COMU as a representative "green" uronium-type amide coupling reagent, ${ }^{47-49}$ was selected as a model process (Scheme 2). We aimed to screen and compare the results of various reaction conditions, including the evaluation of coupling efficiency for different coupling reagents beyond the COMU itself, to reveal the most promising hits in terms of product yield and green chemistry requirements. The choice of aromatic amine $\mathbf{3}$ was dictated by its reduced nucleophilicity in comparison with aliphatic amines, additionally attenuated by an electron-withdrawing ethoxycarbonyl group. We expected that suppressed reactivity of $\mathbf{3}$ in the carbonyl addition reactions would facilitate more reliable differentiation of various coupling conditions. Use of 
phenyl alanine derivative 2 as coupling counterpart provided an additional opportunity to examine the resistance $\alpha$-stereocenter towards its possible epimerization, as commonly encountered in peptide synthesis. ${ }^{9,15}$

\section{Scheme 2. Optimization Experiments}

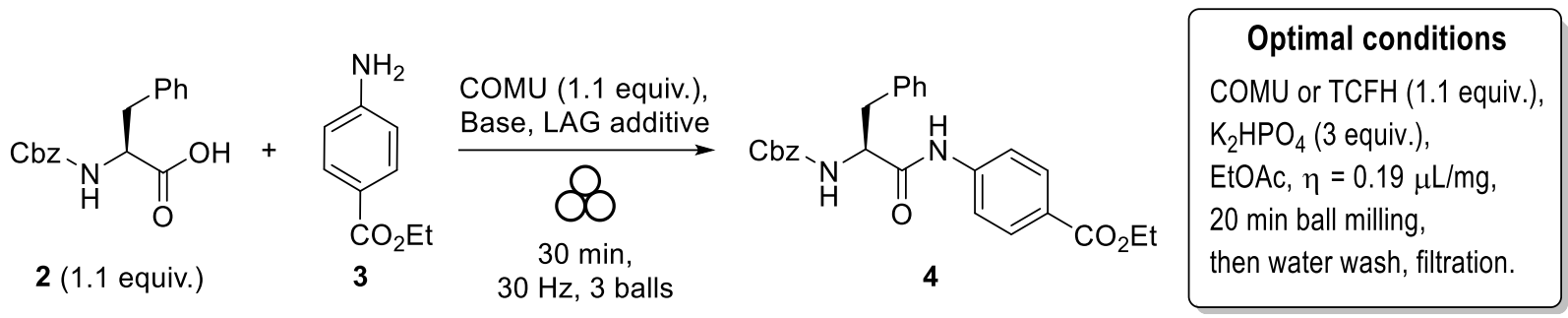

\section{Effect of LAG}

LAG additive $=$ EtOAc, Base $=\mathrm{Na}_{2} \mathrm{CO}_{3}$

$\eta, \mu \mathrm{L} / \mathrm{mg}$

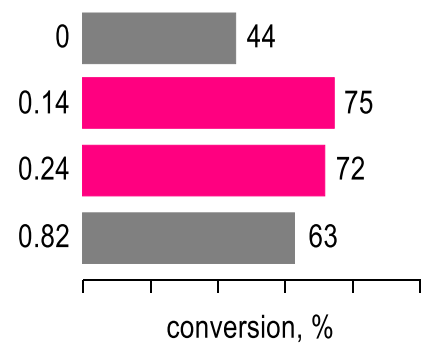

\section{Effect of Base}

$\eta=0.14-0.19 \mu \mathrm{L} / \mathrm{mg}$

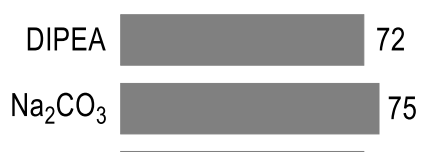

$\mathrm{K}_{3} \mathrm{PO}_{4}$

$\mathrm{K}_{2} \mathrm{HPO}_{4}$

$\mathrm{KH}_{2} \mathrm{PO}_{4}$

$\mathrm{Na}_{2} \mathrm{HPO}_{4}$

$\mathrm{K}_{4} \mathrm{P}_{2} \mathrm{O}_{7}$

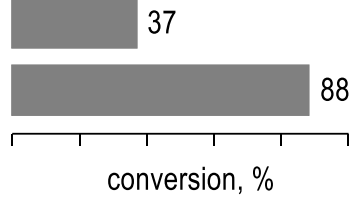

3 Effect of Coupling Reagent

Base $=\mathrm{KH}_{2} \mathrm{PO}_{4}, \eta=0.19 \mu \mathrm{L} / \mathrm{mg}$

EDC 63
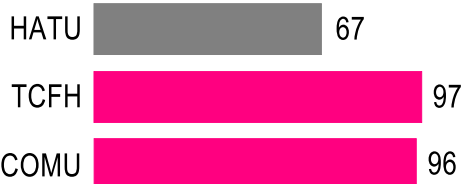

published conditions (ref. 36)

EDC / DMAP $\quad 32$

EDC

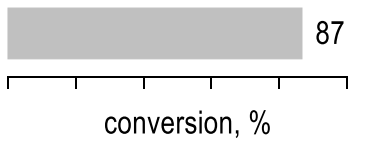

Conversion of 3 into 4 according to ${ }^{1} \mathrm{H}$ NMR analysis of the crude reaction mixtures

The test reactions were run in a Form-Tech Scientific FTS1000 shaker mill operating at $30 \mathrm{~Hz}$ by using $14 \mathrm{~mL}$ zirconia-coated milling jars, $3 \times 7 \mathrm{~mm}$ zirconia milling balls and typical solid reactants loading around $0.3-0.4 \mathrm{~g}$ (including $0.2 \mathrm{mmol}$ of amine 3 as a limiting substrate). After $30 \mathrm{~min}$ milling time, a sample of the crude reaction mixture was treated with $\mathrm{CDCl}_{3}$, followed by separation of insoluble inorganic materials. The conversion of amine $\mathbf{3}$ into amide $\mathbf{4}$ was determined by ${ }^{1} \mathrm{H}$ NMR analysis (see Supporting Information for the details). The amide coupling reagent, base, and amount of liquid additive needed to assist the grinding process (Scheme 2) were identified as the three most crucial parameters affecting the yield of amide $\mathbf{4}$, as described below. 
The addition of a small volume of liquid constitutes an efficient method to enhance the performance of solvent-free mechanochemical reactions, known as liquid-assisted grinding (LAG). ${ }^{22,24}$ The ratio of the volume of liquid $(\mu \mathrm{L})$ added to the amount of solid present $(\mathrm{mg})$ is denoted as $\eta(\mu \mathrm{L} / \mathrm{mg}){ }^{50}$ A value of $\eta=0$ generally corresponds to dry grinding, but in a typical LAG process, $\eta$ is usually between 0 and $1 .{ }^{24}$ Although LAG cannot be described as a totally solvent-free technique, it requires a minimal amount of liquid, especially advantageous if a green solvent is used. Among the latter, ${ }^{20,51,52}$ ethyl acetate appears to be the most promising and chemically compatible candidate to act as a LAG additive in COMU-mediated amide coupling. In our experiments (Scheme 2, Chart 1), the addition of ethyl acetate indeed showed a pronounced effect on the yield of amide $\mathbf{4}$, generated in the mixture of solid reactants $\mathbf{2}$ and $\mathbf{3}$, with COMU reagent and sodium carbonate (ca. 10 equiv.) as a base. Although dry grinding provided a rather modest outcome ( $44 \%$ conversion), LAG resulted in a markedly improved reaction performance, with the optimal $\eta$ value in a range of $0.14-0.24 \mu \mathrm{L} / \mathrm{mg}$, while the further increase of $\eta$ led to slightly diminished conversion values.

The choice of base is also important in amide coupling. State-of-the-art solution approaches commonly apply non-nucleophilic tertiary amines, e.g. $N, N$-diisopropylethylamine (DIPEA). ${ }^{15}$ However, the use of cheap and non-toxic inorganic salts, e.g. $\mathrm{NaHCO}_{3}, \mathrm{~K}_{2} \mathrm{CO}_{3}, \mathrm{NaH}_{2} \mathrm{PO}_{4},{ }^{28,30,33,37}$ insoluble in common organic solvents, can be considered as an additional advantage of mechanochemical reactions. In our hands (Scheme 2, Chart 2), replacement of DIPEA with $\mathrm{Na}_{2} \mathrm{CO}_{3}$ gave similar conversion values (72\% vs $75 \%$ ). For further process optimization, a range of readily available phosphate salts, with notably distinct $\mathrm{p} K_{\mathrm{a}}$ values, were screened. Among them, potassium pyrophosphate $\mathrm{K}_{4} \mathrm{P}_{2} \mathrm{O}_{7}$ and dipotassium phosphate $\mathrm{K}_{2} \mathrm{HPO}_{4}$ provided the best outcomes, especially the latter (96\% conversion). Generally, the performance of phosphate salts does not correlate with Brønsted basicity of the respective anions. Although the poor outcome with $\mathrm{KH}_{2} \mathrm{PO}_{4}$ (only $18 \%$ conversion) in comparison with $\mathrm{K}_{2} \mathrm{HPO}_{4}(96 \%)$ could be probably connected with the significantly reduced base strength of the former (respective $\mathrm{p} K_{\mathrm{a}}$ values 2.12 vs $7.21 ; \mathrm{p} K_{\mathrm{a}}$ of $\mathrm{RCO}_{2} \mathrm{H}$ is typically about $4-5$ in aqueous media), ${ }^{53}$ much more basic $\mathrm{K}_{3} \mathrm{PO}_{4}\left(\mathrm{p} K_{\mathrm{a}} 12.32\right)$ also afforded amide 4 with reduced efficiency (72\%). Surprisingly, the counter-cation effect $\left(\mathrm{Na}^{+}\right.$vs $\mathrm{K}^{+}$) also had a prominent impact on reaction outcome ( $37 \%$ vs $96 \%$, for $\mathrm{Na}_{2} \mathrm{HPO}_{4}$ and $\mathrm{K}_{2} \mathrm{HPO}_{4}$ respectively). These results clearly indicate that the effect of an inorganic base on a solid-state reaction is more intricate than trivial proton transfer. 
Finally, amide coupling reagents are essential for attaining high yields. The selection of coupling reagent was governed by considering chemical (substrate scope, reactivity); safety; and environmental issues. Uronium salts are advantageous because of their prominent reactivity and efficient reaction rates, ${ }^{8,14}$ but the most commonly applied triazole-based reagents, such as HBTU and HATU, possess dangerous explosive properties ${ }^{54}$ and pose significant health risks. ${ }^{55} \mathrm{COMU}$ was introduced as a safe and "greener" replacement. ${ }^{47,48,56}$ To our delight, COMU also noticeably exceeded the coupling efficiencies of HATU and EDC in our experiments (Scheme 2, Chart 2), delivering a high $96 \%$ conversion. TCFH can be considered as an even more reactive alternative with better atom economy, affording a high $97 \%$ yield of amide 4 within only $10 \mathrm{~min}$.

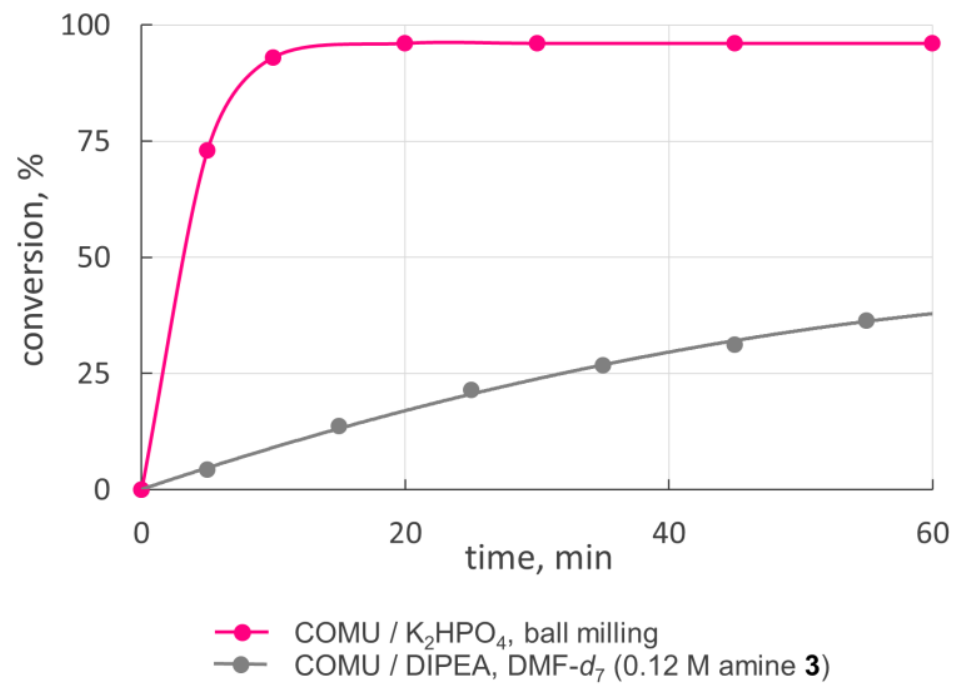

Figure 1. Accumulation of amide 4 over time in mechanochemical (purple) and solvent-based (gray) reactions.

The mechanochemical amidation with $\mathrm{COMU} / \mathrm{K}_{2} \mathrm{HPO}_{4}$ was also rapid, reaching the maximal conversion within 20 min (Figure 1; see the Supporting Information for further detail), far surpassing the rate of the solution-based process (in DMF- $d 7$, Figure 1). The latter reached the maximal 70\% conversion after approximately $20 \mathrm{~h}$ (see the Supporting Information). Concurrently, about $30 \%$ of COMU reagent degraded due its well-known hydrolytic instability in DMF solutions, which is often referred as the main disadvantage of COMU. ${ }^{57,58}$ Evidently, this drawback can be fully eliminated under solvent-free conditions.

After achieving these results in the optimization experiments, we formulated the optimal experimental procedure as follows: COMU or TFCH (1.1 equiv.) as coupling reagents; $\mathrm{K}_{2} \mathrm{HPO}_{4}$ (3 
equiv.) as base; ethyl acetate as LAG additive, and 20 min milling time. The amount of solid base (3 equiv.) was adjusted to keep $\eta$ within the optimal range $(\sim 0.2 \mu \mathrm{L} / \mathrm{mg})$, but not less than 2 equiv. required according to the reaction stoichiometry. Furthermore, an additional equivalent of $\mathrm{K}_{2} \mathrm{HPO}_{4}$ was required to release free amine when ammonium salt was used as the starting material. Isolation of pure amide 4 was achieved with a high $96 \%$ yield by simple water wash and filtration since all by-products are water soluble. No detectable racemization of the chiral center in $\mathbf{4}$ occurred during the synthesis, as was established by the chiral phase HPLC chromatography (see the Supporting Information).

Green Chemistry Metrics Comparison. The advantages and drawbacks of the developed mechanochemical amidation methods were further revealed and compared with the solution-based reaction by analyzing the respective green metrics (Table 1). The metrics were calculated and assessed by marking them with red, orange, or green flags by following the Clark's unified metrics toolkit (see Supporting Information). ${ }^{59}$ Atom economy (AE), reaction mass efficiency (RME), and process mass intensity (PMI) are defined as follows: ${ }^{59}$

$$
\begin{gathered}
\mathrm{AE}=\frac{\text { molecular weight of product }}{\text { total molecular weight of reactants }} \times 100 \\
\mathrm{RME}=\frac{\text { mass of isolated product }}{\text { total mass of reactants }} \times 100 \\
\mathrm{PMI}=\frac{\text { total mass in a process }}{\text { mass of product }}
\end{gathered}
$$

First, isolated yields and product purity were much better in mechanochemical reactions, due to the higher conversion and more facile isolation procedure discussed above. Atom economy was a bit higher for the TCFH-mediated reaction because of lower molecular weight of TCFH. RME reflects both product yield and atom economy issues and was lower for the solution-based reaction. Comparison of PMI values clearly shows that mechanochemical reactions produce far less waste. Excluding mass-extensive work-up procedures, solvent occupied 84\% of PMI for the solutionbased reaction and only about $15 \%$ (LAG additive) for the mechanochemical conditions. Furthermore, sustainable solvents like water and ethyl acetate were used in the latter, in contrast with toxic DMF. 
Table 1. Comparison of Green Metrics for Mechanochemical and Solution-Based Amidation Reactions

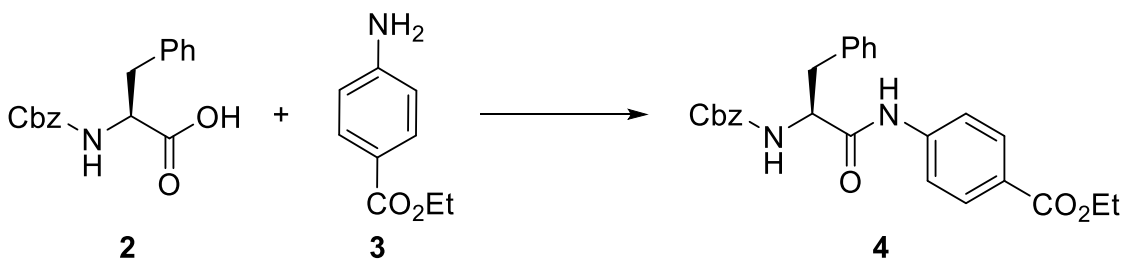

\begin{tabular}{|c|c|c|c|}
\hline \multirow{2}{*}{ Metrics } & \multicolumn{2}{|c|}{ Mechanochemistry } & \multirow{2}{*}{$\begin{array}{c}\text { Solution }^{a} \\
\text { COMU / DIPEA in DMF }\end{array}$} \\
\hline & $\mathrm{COMU} / \mathrm{K}_{2} \mathrm{HPO}_{4}$ & TCFH / K ${ }_{2} \mathrm{HPO}_{4}$ & \\
\hline Yield $(\%)^{b}$ & 96 & 92 & $70 \mathrm{~F}$ \\
\hline Atom economy $(\%)^{c}$ & 50 & 60 & 50 \\
\hline RME (\%) & 46 & 53 & 35 \\
\hline PMI (total), including: & 196.3 & 203.7 & 1464.7 \\
\hline PMI (reactants) & 3.4 & 3.1 & 3.7 \\
\hline PMI (solvent) & 0.6 & 0.6 & 18.9 \\
\hline PMI (work-up) & 192.3 & 200 & $1442.2^{d}$ \\
\hline Solvent choice & EtOAc, water & EtOAc, water & DMF \\
\hline Work-up, isolation & filtration & filtration & chromatography \\
\hline Health and safety & & & \\
\hline Main hazard statements ${ }^{e}$ & H302, H312 & H360 & $\mathrm{H} 226,312,332,360$ \\
\hline
\end{tabular}

To determine the safety risks, a combination of physical, health, and environmental threats must be assessed, which can be done with the help of $\mathrm{MSDS}^{60}$ and further available safety data. ${ }^{54} \mathrm{DMF}$, for instance, is a flammable $(\mathrm{H} 226)$, acute toxic $(\mathrm{H} 312,332)$, as well as a reproductive toxin $(\mathrm{H} 360)$ and can thus be cited as the main hazard contributor for the solution-based process, which therefore received a red flag. For the mechanochemical reactions, the TCFH-mediated process was given a red flag due to the production of tetramethylurea by-product (reproductive toxin, H360). On the other hand, exothermic decomposition with a thermal onset of $127^{\circ} \mathrm{C}$ can be considered as the main hazard of COMU, according to a recent study. ${ }^{54}$ However, this property produced an orange flag, since COMU-mediated mechanochemical amidation protocol operates at room temperature. To conclude, although the developed mechanochemical amidation conditions cannot be considered totally safe, the risks are minimal because of its room temperature operation and relatively low 
amount of produced waste, as opposed to the solution-based reaction (see Supporting Information for additional safety considerations).

Substrate Scope for mapping reactivity with COMU and TCHF. Having established the optimal conditions, substrate scope and limitations was briefly examined on a range of amine and acid coupling partners (Scheme 3 ).

Scheme 3. Substrate Scope for Mechanochemical Amidation with $\mathrm{COMU} / \mathrm{K}_{2} \mathrm{HPO}_{4}$ and $\mathrm{TCFH} / \mathrm{K}_{2} \mathrm{HPO}_{4}$ Systems.
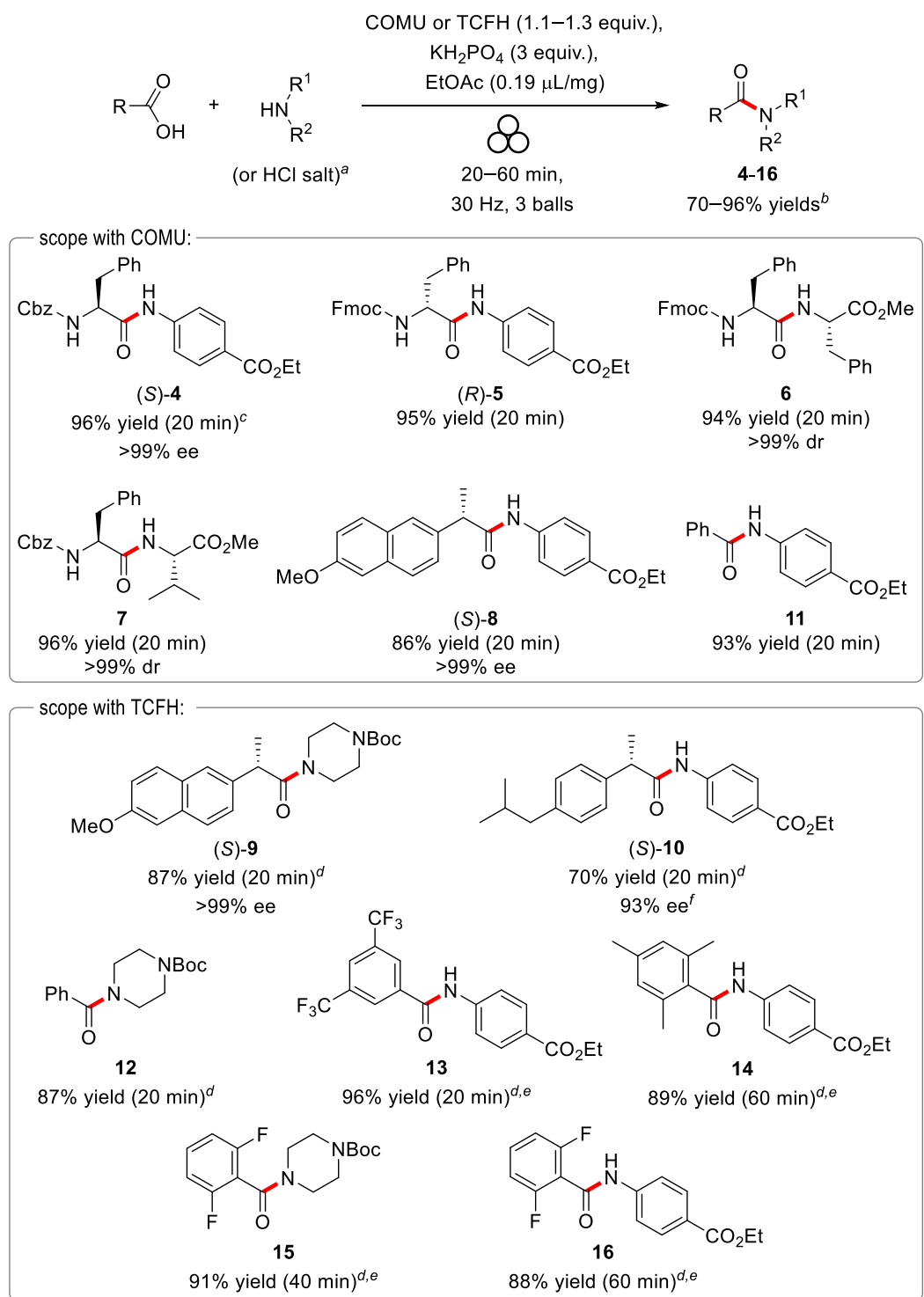

${ }^{a}$ Amine hydrochloride salt was used for preparation of peptides 6 and 7. ${ }^{b}$ Yields of isolated products ${ }^{c}$ Obtained in $92 \%$ yield and $>99 \%$ ee with TCFH. ${ }^{d}$ Isolated by column chromatography. ${ }^{e}$ With 1.3 equiv. of TCFH. ${ }^{f} 94 \%$ ee with COMU. 
The substrate scope included, besides other, $\mathrm{N}$ - and C-protected amino acids and pharmaceutically relevant starting materials (e.g. (S)-naproxen, $(S)$-ibuprofen, benzocaine 3, $N$-Boc-protected piperazine). In addition to the foremost example of Cbz-masked amide $(S)-4$ comprehensively described above, its Fmoc-protected analogue $(R)-5$ was obtained in a high $95 \%$ yield by using the COMU-mediated reaction. Following the same protocol, dipeptides 6 and 7 with sterically hindered amino acid residues (phenylalanine and valine) were flawlessly prepared in high yields. No detectable epimerization of the stereocenters was noted in these cases. Coupling of $(S)-(+)-6-$ methoxy- $\alpha$-methyl-2-naphthaleneacetic acid [(S)-naproxen] with amine $\mathbf{3}$ provided a more demanding test for stereochemical integrity, since 2-arylpropionic acids are prone to easy epimerization. ${ }^{61-64}$ The amide product $(S)$-8 was obtained from $(S)$-naproxen with a high $86 \%$ yield and excellent stereochemical purity (>99\% ee). This was also the case in the TCFH-mediated reaction, which showed high reactivity and a subtle amount of epimerization for $(S)$-9. On the other hand, amidation of $(S)$-ibuprofen (98\% ee) produced $(S)$-10 with slightly degraded optical purity (93-94\% ee). Crude amides 10, 12, 15 appeared as oils immediately following the milling, which eventually enabled a chromatographic isolation for these cases (see Supporting Information).

One advantage of TCFH over COMU-mediated amide coupling is the higher reactivity of the former reagent, which makes it more suitable for less reactive substrates. This property was explicitly revealed during the amidation of sterically hindered ortho-substituted benzoic acids. Thus, coupling of benzoic acid with benzocaine 3 proceeded well under the COMU-mediated protocol, furnishing amide 11 in a 93\% yield after $20 \mathrm{~min}$ of milling time. Conversely, 2,4,6trimethylbenzoic acid under the same conditions produced only $22 \%$ of the target amide $\mathbf{1 4}$, without any further improvement, even when a longer milling time (up to $60 \mathrm{~min}$ ) was applied. After the brief optimization studies (see Supporting Information), we found that a slight excess (1.3 equiv.) of more reactive TCFH and at least $60 \mathrm{~min}$ of milling time are required to attain a high $89 \%$ yield of 14. Moreover, chromatographic purification of $\mathbf{1 4}$ was necessary to separate mesitoic anhydride impurity. Diminished reactivity was also observed for 2,6-difluorobenzoic acid, furnishing amides 15 and 16 in reactions with $N$-Boc piperazine and low-nucleophilic amine 3 in acceptable yields after milling times of 40 and $60 \mathrm{~min}$, respectively. On the other hand, coupling of the same amines with benzoic and 3,5-bis(trifluoromethyl)benzoic acids proceeded flawlessly, producing amides $\mathbf{1 2}$ and $\mathbf{1 3}$ with excellent yields and brief reaction times. 
Activating Effect of Phosphate Salts. During the optimization studies, the enhancement of yields with dipotassium phosphate and potassium pyrophosphate was especially notable (Scheme 2, Chart 2). We speculated that phosphate salts could additionally contribute to the activation of the carboxyl substrate $\mathbf{2}$ via the formation of acyl phosphate intermediates containing a "high-energy" phosphoester bond, prone to easy nucleophilic amine attack. ${ }^{65,66}$ Interestingly, the same pathway is also involved in the ATP-dependent biosynthesis of amide bond-containing biomolecules. ${ }^{11}$ The plausibility of our assumption is further supported by existing literature showing that acyl phosphates can be indeed generated in solution by the DCC-mediated coupling of carboxylic acids with phosphate salts. ${ }^{67-69}$ To confirm the credibility of our hypothesis, mechanochemical synthesis of acyl phosphates from carboxylic acids and phosphate salts, mediated by COMU and TCFH, was attempted.

As expected, a 20-min ball milling of COMU (1.1 equiv.) with acetic acid (1 equiv.) and $\mathrm{K}_{2} \mathrm{HPO}_{4}$ (3 equiv.) yielded $60 \%$ of acetyl phosphate 17, which was confirmed by NMR analysis of the freshly obtained reaction mixture in $\mathrm{D}_{2} \mathrm{O}$ solution (Figure 2). Acetyl phosphate 17 displayed a singlet signal at $\delta=-2.1 \mathrm{ppm}$ in ${ }^{31} \mathrm{P} \mathrm{NMR}$, which rapidly disappeared after the addition of morpholine, both in $\mathrm{D}_{2} \mathrm{O}$ solution and in the solid state (see Supporting Information). In the ${ }^{13} \mathrm{C}$ NMR spectrum, carbonyl group 17 showed a doublet signal at $\delta=168.1 \mathrm{ppm}\left(J_{\mathrm{CP}}=8.8 \mathrm{~Hz}\right)$, due to its coupling with the neighboring phosphorus. ${ }^{65}$ Significantly lower yields of $\mathbf{1 7}$ were attained with $\mathrm{K}_{3} \mathrm{PO}_{4}$ or with TCFH as coupling reagent (Figure 2). The reaction of acetic acid with $\mathrm{K}_{4} \mathrm{P}_{2} \mathrm{O}_{7}$ produced acetyl pyrophosphate $\mathbf{1 8}$ in a $50 \%$ yield, according to ${ }^{31} \mathrm{P}-\mathrm{NMR}$ analysis. As a result of the non-equivalence of phosphorus atoms in 18, a pair of doublet signals appeared in ${ }^{31} \mathrm{P}$ $\mathrm{NMR}$, at $\delta=-5.0$ and $-17.9 \mathrm{ppm}\left(d, J_{\mathrm{PP}}=21.7 \mathrm{~Hz}\right)$, thus confirming its structure. ${ }^{67}$ As an extra example, the generation of acyl phosphate $19\left(50 \%\right.$ yield, $\delta=-7.6 \mathrm{ppm}$ in $\left.{ }^{31} \mathrm{P} \mathrm{NMR}\right)$ was also successful from Cbz-masked phenyl alanine 2, which was similar to the acetic acid outcome. 

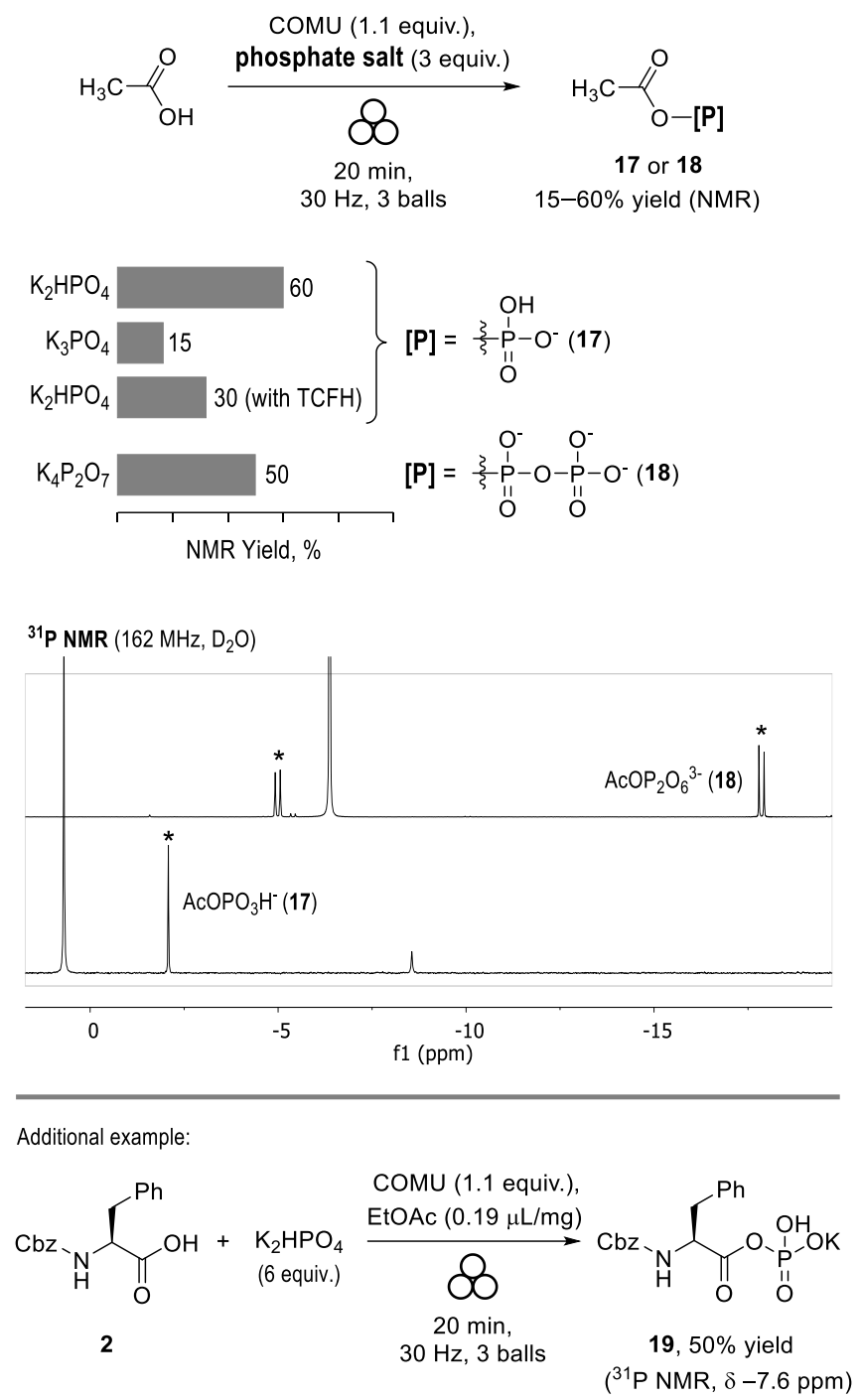

Figure 2. Mechanochemical generation of acyl phosphates 17, 19 and acetyl pyrophosphate 18. Signals of 17 and 18 in the traces of ${ }^{31} \mathrm{P}$ NMR spectra are marked with asterisks. Other signals belong to inorganic phosphates (see Supporting Information).

These results clearly indicate that generation of acyl phosphates can indeed take place in these newly developed mechanochemical amidation approaches and could account for the observed enhanced efficiency of $\mathrm{K}_{2} \mathrm{HPO}_{4}$ and $\mathrm{K}_{4} \mathrm{P}_{2} \mathrm{O}_{7}$ additives. To evaluate the contribution of the acyl phosphate pathway (Scheme 4, via intermediate $\mathbf{C}$ ) against the manifested activated ester pathway (via intermediates $\mathbf{A}$ and $\mathbf{B}),{ }^{48,70}$ the following experiment was undertaken. Amide coupling reaction of potassium salt of $\mathbf{2}$ with amine $\mathbf{3}$ without the phosphate salt additive afforded amide $\mathbf{4}$ in $80 \%$ yield, $16 \%$ lower than that obtained with $\mathrm{K}_{2} \mathrm{HPO}_{4}$. It was concluded from these results that in the amidation reaction leading to $4, \mathrm{~K}_{2} \mathrm{HPO}_{4}$ acts primarily as a base performing deprotonation 
of 2 , but it also contributes at least $16 \%$ to the formation of amide 4 via acyl phosphate 19. This estimation correlates well with the results of other optimization experiments (Scheme 2, Chart 2). For example, $\mathrm{K}_{3} \mathrm{PO}_{4}$ produced a rather low $15 \%$ yield of acetyl phosphate 17 (Figure 2), which also agrees with the lower conversion to amide $\mathbf{4}$ in the comparison with $\mathrm{K}_{2} \mathrm{HPO}_{4}$. The acyl phosphate pathway probably contributes less in the case of the more reactive TCFH reagent, which also produced a rather low $30 \%$ yield of $\mathbf{1 7}$ (Figure 2). The exact mechanistic sequence leading to acyl phosphates $\mathbf{C}$ from $\mathrm{COMU}, \mathrm{RCO}_{2} \mathrm{H}$ and $\mathrm{K}_{2} \mathrm{HPO}_{4}$ remains unclear but may include the reaction of acyl uronium intermediate $\mathbf{A}$ with $\mathrm{HPO}_{4}{ }^{2-}$ anion (Scheme 4) or, alternatively, the initial formation of uronium phosphate ${ }^{71}$ by the reaction of COMU with $\mathrm{K}_{2} \mathrm{HPO}_{4}$.

\section{Scheme 4. Plausible Mechanistic Pathways Leading to Amide Product}
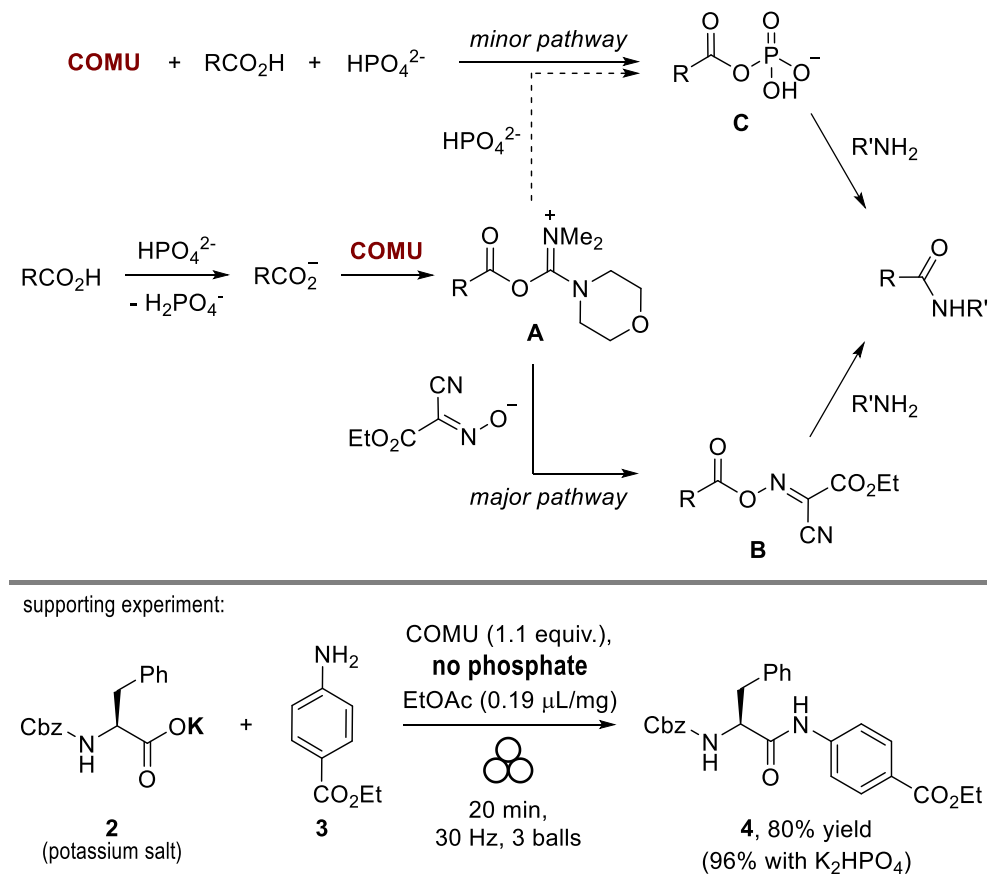

Challenging Amide Bond Formation. As shown above, the coupling of low nucleophilic amine 3 with sterically hindered mesitoic acid could be efficiently mediated by the $\mathrm{TFCH} / \mathrm{K}_{2} \mathrm{HPO}_{4}$ reagent system (Scheme 3). In accordance with existing literature, ${ }^{61,72}$ we selected the coupling of electrondeficient 4-aminobenzonitrile 20 with 2-methyl-2-phenylpropanoic acid 21 (Scheme 5), an even more arduous way to test the performance of mechanochemical amidation protocols. Brief screening of various coupling conditions was undertaken, and conversion to amide product 22 was determined by ${ }^{1} \mathrm{H}$ NMR analysis after 60 min of milling time (Scheme 5). 
Scheme 5. Mechanochemical Coupling of Hindered Carboxylic Acids and Poor Nucleophilic Amines
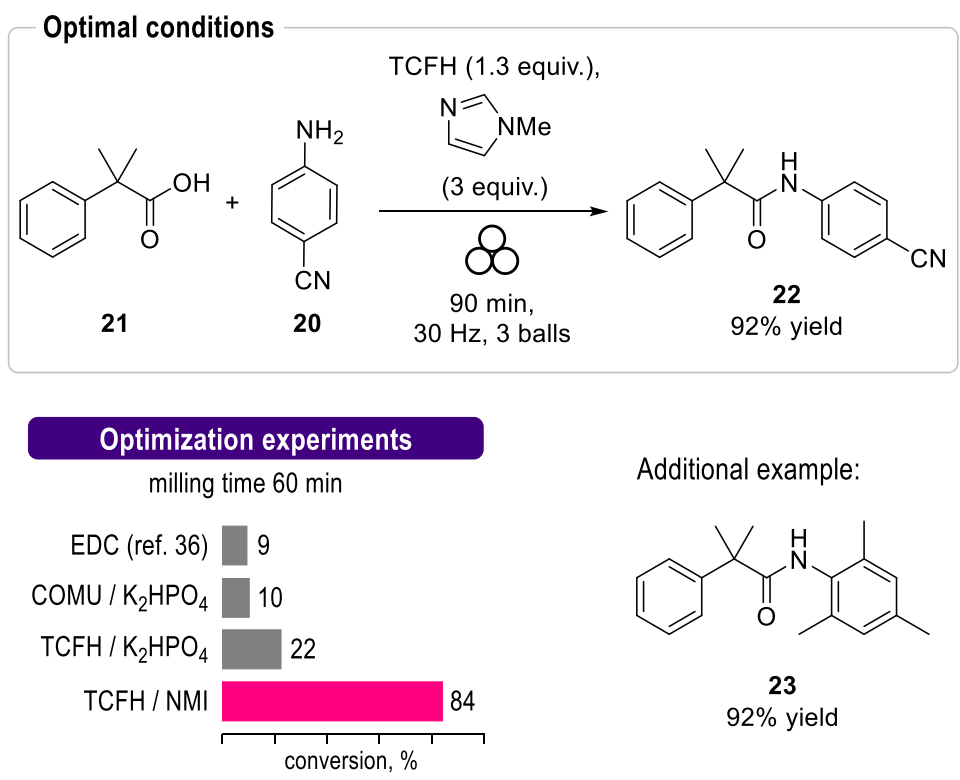

Additional example:

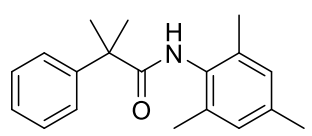

$92 \%$ yield
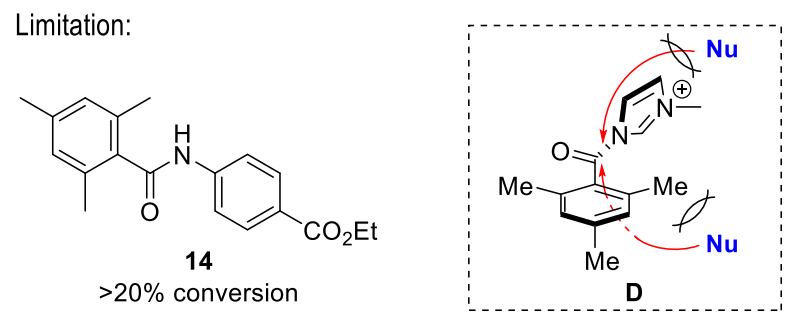

The use of EDC alone, ${ }^{36}$ or the $\mathrm{COMU} / \mathrm{K}_{2} \mathrm{HPO}_{4}$ system, yielded only a low $\sim 10 \%$ conversion. Combination of TCFH and $\mathrm{K}_{2} \mathrm{HPO}_{4}$ delivered a noticeably better outcome but still failed to raise the conversion above $22 \%$. According to the recent study of Beutner et al., ${ }^{61} \mathrm{~N}$-methylimidazole (NMI) and TCFH reagent combined provided a high yield of 22 in solution, due to in situ generation of reactive $N$-acyl imidazolium ions. To our gratification, same combination of reagents also worked well under the solvent-free conditions, affording respectable $84 \%$ conversion after a 60-min reaction time. Finally, a slight excess (1.3 equiv.) of TCFH reagent, along with a bit longer milling time (90 min), allowed us to obtain pure amide 22 in $92 \%$ isolated yield after an aqueous work-up (see Supporting Information). Following the same reaction protocol, the coupling of 21 with sterically hindered 2,4,6-trimethylaniline was performed and furnished the corresponding amide 23 with a $92 \%$ yield. Notably, high yields of amides 22,23 were attained in a rather efficient reaction time of $1.5 \mathrm{~h}$, in significant contrast with the solution-based reaction ( $21 \mathrm{~h}$ for amide 22). ${ }^{61}$ 
Surprisingly, the same highly reactive combination of reagents failed to render amide $\mathbf{1 4}$ from mesitoic acid with yields exceeding 20\%. This was also the case in the $\mathrm{CD}_{3} \mathrm{CN}$ solution (see Supporting Information). We found that the reaction was stopped due to the formation of sterically bulky and therefore non-planar $N$-acyl imidazolium $\mathbf{D}$, which, in contrast to the analogous species produced from benzoic acid, was totally inert towards the subsequent reaction with amine $\mathbf{3}$ (see Supporting Information for further detail). Inertness of $\mathbf{D}$ could be explained by the efficient steric shielding of the carbonyl group with both neighboring mesityl and imidazolyl moieties, preventing attack of a nucleophile along the Bürgi-Dunitz trajectory (Scheme 5). This stands in sharp contrast to the successful $\mathrm{TCFH} / \mathrm{K}_{2} \mathrm{HPO}_{4}$-mediated transformation, where the less sterically crowded intermediate species are expected to form (e.g. mesitoyl chloride, uronium or phosphate).

Amide Coupling of Biotin[6]uril. As a part of our ongoing efforts towards the development of new chiral supramolecular receptors, ${ }^{38-43}$ we needed an expedient synthetic procedure for derivatization of biotin[6]uril (1), ${ }^{44}$ easily available in multigram quantities by HCl-catalyzed condensation of formaldehyde with D-biotin. The starting macrocyclic molecule, notable for its anion binding properties, common for the cucurbituril family, ${ }^{73-77}$ satisfies 6 carboxylic functions, which could be conveniently coupled with various amines, thus providing facile access to a library of diversely functionalized chiral macrocyclic receptors. Although amide coupling of carboxylates in 1 might appear simple, unencumbered by any steric or electronic influence, full amidation of $\mathbf{1}$ is challenging because it proceeds via six consecutive steps. For example, if a high $97 \%$ yield were produced during each step, the fully functionalized product would eventually generate only a $(0.97)^{6} \cdot 100 \%=83 \%$ yield, while the rest of the produced material would contain a set of "failed" underfunctionalized molecules, thus necessitating time-consuming, laborious and mass-inefficient chromatographic purification. The situation resembles the synthesis of oligopeptides and oligonucleotides, in which an extremely high coupling efficiency (>99\% per coupling step) is required to attain reasonable yields and high purity of long-chain oligomers, and it is customarily achieved by using an excess of highly reactive coupling reagents. ${ }^{56}$ The low solubility of $\mathbf{1}$ in the environmentally benign and volatile organic solvents, compatible with the conventional amidation protocols (e.g. ethyl acetate), constitutes an additional restriction of the solution-based chemistry. We believed that the high coupling efficiency observed under the solvent-free conditions would allow us to perform the desired functionalization in a high-yielding, and scalable manner without using an excess of reagents, toxic solvents, or laborious purification. 
As a convenient model reaction for this study, we selected the amide coupling of $\mathbf{1}$ with methyl ester of phenyl alanine $\mathbf{2 4}$ (used as $\mathrm{HCl}$ salt, see Scheme 6). At its outset, this task required us to explore the performance of different amide coupling conditions. Only a slight excess of amine $\mathbf{2 4}$ and a coupling reagent (7-7.8 equiv., which is 1.16-1.3 equiv. per $\mathrm{CO}_{2} \mathrm{H}$ group of $\mathbf{1}$ ) were applied in the optimization experiments. It was expected that more reactive combinations of reagents would deliver higher yields of the hexa-amide product 25. Based on our previous findings, the order of coupling efficiency for the different reagent systems can be roughly plotted as follows: EDC $\mathrm{COMU} / \mathrm{K}_{2} \mathrm{HPO}_{4}<\mathrm{TCHF} / \mathrm{K}_{2} \mathrm{HPO}_{4}<<\mathrm{TCHF} / \mathrm{NMI}$. Although such generalizations must be made with care since the coupling performance is substrate-dependent ${ }^{8,78}$ and exceptions are possible (e.g. case of amide $\mathbf{1 4}$ above), this preliminary reactivity plot provided a helpful guide.

Scheme 6. Derivatization of biotin[6]uril (1) via six-fold amidation with L-phenylalanine methyl ester (24).
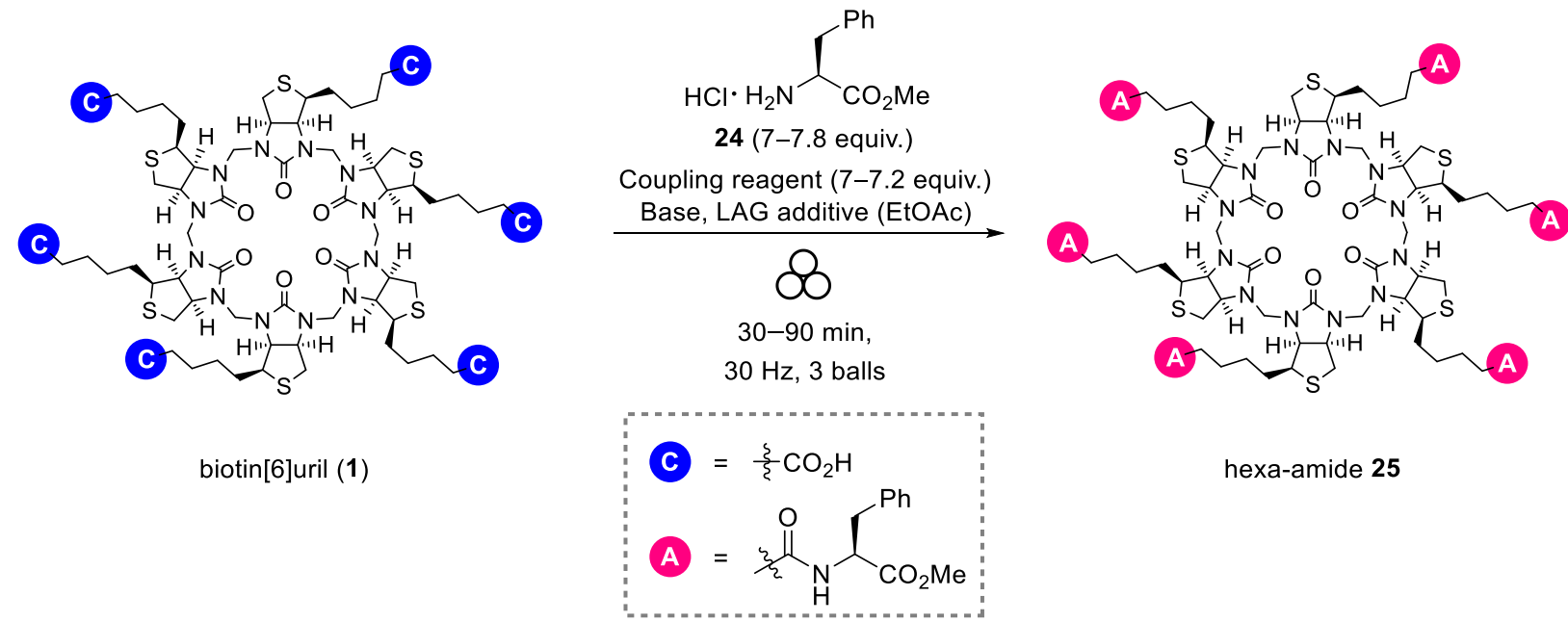

hexa-amide 25

Outcomes of the test reactions were analyzed by HPLC (Figure 3, see Supporting Information for further detail) and quantified by calculating HPLC area percentage for the hexa-amide product $\mathbf{2 5}$ (Srel, Table 2), relative to underfunctionalized compounds.

Table 2. Amide coupling of biotin[6]uril with phenylalanine methyl ester 24

\begin{tabular}{|c|c|c|c|c|c|c|}
\hline Entry & & Reaction conditions $^{a}$ & $\begin{array}{l}\text { Liquid chemicals } \\
\text { (additives, solvents) }\end{array}$ & $\begin{array}{c}\eta, \\
\mu \mathrm{L} / \mathrm{mg}\end{array}$ & $\begin{array}{l}\text { Time, } \\
\min \end{array}$ & $\begin{array}{l}S_{r e l}, \\
\%^{b}\end{array}$ \\
\hline 1 & Ball milling & $\mathrm{COMU} / \mathrm{K}_{2} \mathrm{HPO}_{4}$ & EtOAc & 0.19 & 90 & 16 \\
\hline 2 & & $\mathrm{TCFH} / \mathrm{K}_{2} \mathrm{HPO}_{4}$ & EtOAc & 0.19 & 90 & 16 \\
\hline 3 & & $\mathrm{EDC}^{-} \mathrm{DMAP}^{c}$ & $\mathrm{CH}_{3} \mathrm{NO}_{2}$ & 0.25 & 90 & 55 \\
\hline
\end{tabular}




\begin{tabular}{|c|c|c|c|c|c|c|}
\hline 4 & & TCFH / NMI & NMI & 0.29 & 90 & 52 \\
\hline 5 & & $\mathrm{TCFH} / \mathrm{NMI}^{d}$ & NMI & 0.32 & 60 & 86 \\
\hline 6 & & $\mathrm{TCFH} / \mathrm{NMI}^{d} / \mathrm{NaCl}^{e}$ & NMI & 0.16 & 60 & 73 \\
\hline 7 & & $\mathrm{TCFH} / \mathrm{NMI}^{d}$ & NMI, DMF & 0.53 & 60 & 97 \\
\hline 8 & & $\mathrm{TCFH} / \mathrm{NMI}^{d}$ & NMI, heptane & 0.64 & 60 & 84 \\
\hline 9 & & $\mathbf{T C F H} / \mathbf{N M I}^{d}$ & NMI, EtOAc & 0.64 & 60 & 98 \\
\hline 10 & Slurry stirring & TCFH / NMI ${ }^{d}$ & NMI, EtOAc & 0.64 & 60 & 95 \\
\hline 11 & Solution (DMF) & HATU, DIPEA & DIPEA, DMF & 2.1 & 60 & 68 \\
\hline 12 & & $\mathrm{TCFH}, \mathrm{NMI}^{d}$ & NMI, DMF & 2.4 & 60 & 98 \\
\hline
\end{tabular}

${ }^{a}$ Reaction conditions: biotin[6]uril (50-70 mg, 0.03-0.05 mmol), 24 (7 equiv.), coupling reagent (7 equiv.), base (18 equiv.), unless other specified. ${ }^{b}$ HPLC area percentage of hexa-amide 25 , relative to other amide products. ${ }^{c} 12$ equiv. of DMAP was used, following the published procedure, see ref. ${ }^{36} .{ }^{d}$ With 7.2 equiv. of TCFH, 7.8 equiv. of 24 and 21 equiv. of NMI. ${ }^{e} \mathrm{NaCl}$ was used as grinding additive.

These initial experiments (Table 2, entries 1-4) clearly indicated that complete hexafunctionalization of $\mathbf{1}$ is difficult to perform. Thus, both the COMU and $\mathrm{TCFH} / \mathrm{K}_{2} \mathrm{HPO}_{4}$ systems produced a mixture of phenylalanine-derivatized biotin[6]urils, containing all possible products from mono to hexa-amide $\mathbf{2 5}$, the latter displaying a rather low $16 \%$ contribution (entries 1 and 2; Figure 3A). The use of EDC/DMAP combination (entry 3), ${ }^{36}$ was more successful in this case, primarily producing a mixture of penta- and hexa-amides (Figure 3B). The highly reactive TCFH/NMI combination (entry 4) generated hexa-amide $\mathbf{2 5}$ as its main reaction product, but it was noticeably contaminated with underfunctionalized compounds (52\% HPLC area, Figure 3C). 


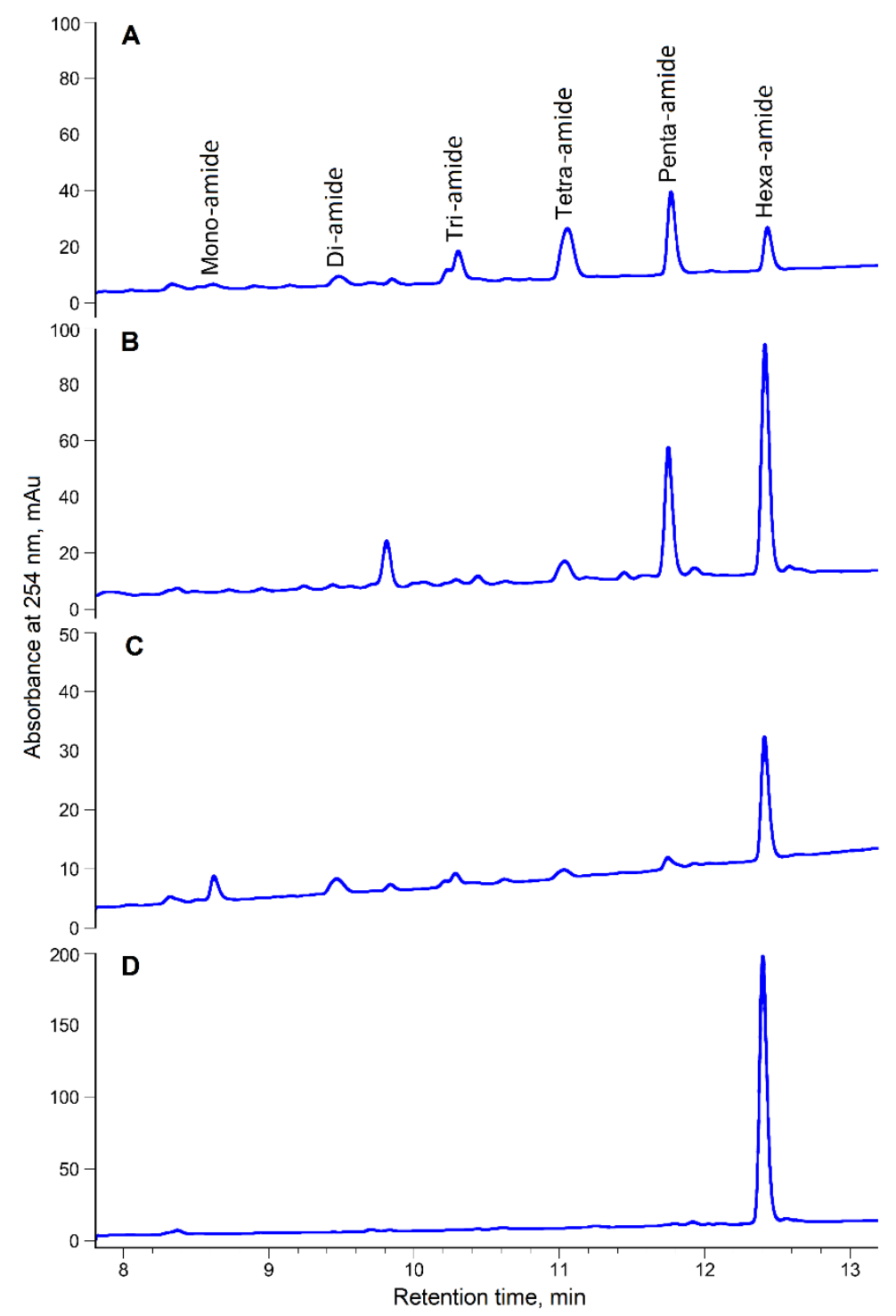

Figure 3. Derivatization of biotin[6]uril via amide coupling with phenylalanine methyl ester 24. Traces of HPLC chromatograms for the selected reaction mixtures: A) $\mathrm{COMU} / \mathrm{K}_{2} \mathrm{HPO}_{4}$; B) EDC/DMAP; C) TCFH/NMI; D) TCFH/NMI with EtOAc as LAG additive.

Notably, at least 90-min milling times had to be applied, since samples taken after 30 and 60 min still showed incomplete conversion (see the Supporting Information). Although the FTS1000 shaker mill could hypothetically achieve long milling, we considered any time longer than $1.5 \mathrm{~h}$ as impractical; therefore, our next goal was to adjust the reaction parameters accordingly, in order to reach at least $90 \%$ conversion within a $1.5-\mathrm{h}$ reaction time.

Applying a slightly greater excess of TFCH (1.2 equiv. per carboxylate) and NMI (3.5 equiv. per carboxylate) noticeably improved the yield of the target product, 25 (86\% HPLC area, entry 5), 
and also shortened the reaction time. For further improvement, a screening of optimal $\eta$ and LAG additive was performed. Since NMI is a liquid, and liquid tetramethylurea is produced, the addition of solid $\mathrm{NaCl}$ was attempted to reduce the initial $\eta$ to $0.16 \mu \mathrm{L} / \mathrm{mg}$. However, this distinctly reduced the yield of the product (73\% HPLC area, entry 6). On the other hand, the addition of a few drops (ca. 35-50 $\mu \mathrm{L}$ ) of solvents noticeably improved the outcome (entries 7 to 9) and was best when polar solvents like DMF or EtOAc were added (entries 7 and 9). These results clearly indicate that the nature of LAG additive plays an important role $^{37}$ and can substantially increase reaction rate, a probable result of the favorable interactions of the polar reactants with the mobile surface layers of LAG additive and improved mass transfer. ${ }^{24}$ The outcome with EtOAc was especially remarkable, providing 25 with the best purity (98\% HPLC area, Figure 3D). Since the reaction mixture visibly liquefied as the reaction progressed (due to the generation of tetramethylurea), slurry stirring was also tried instead of the ball milling (entry 10) and resulted in slightly reduced coupling efficiency. Solution-based amide couplings were performed in DMF (entries 11 and 12) for the comparison with mechanosynthesis. Homogeneous solutions were obtained with an amount of solvent (ca. 0.5 $\mathrm{mL}$ ) comparable to the weight of solid reactants (ca. $0.24 \mathrm{~g}$ ), what kept $\eta$ at around $2 \mu \mathrm{L} / \mathrm{mg}$. In the DMF solution, HATU was another frequently used and highly reactive uronium-based amide coupling reagent that produced a rather modest outcome (entry 11). Conversely, the coupling efficiency of the TCFH/NMI combination in DMF solution (entry 12) was virtually the same as in the DMF-free transformation to a solid state (entry 9). Importantly, a bulk amount of harmful solvent was fully avoided in the latter.

Under the optimal reaction conditions (entry 9), the desired hexa-amide product 25 was isolated in a nearly quantitative yield and 95\% HPLC-purity (relative to all other peaks) after the simple water wash and filtration. Purity of product was further increased (99\% according to HPLC) by following the simple purification protocol (filtration of chloroform solution via Celite ${ }^{\circledR}$, and then precipitation with hexane from EtOAc solution [see Supporting Information]). The same amide coupling reaction was also successful at loadings that were 3 times higher (150 mg of 1 per milling jar, 300 mg total), creating 25 in 80\% isolated yield and 99\% HPLC purity, albeit with a longer milling time (90 $\mathrm{min})$. 


\section{CONCLUSIONS}

In conclusion, we have developed a new mechanochemical approach for the direct synthesis of amides from carboxylic acids and amines by employing uronium-type amide coupling reagents (COMU, TCFH) and $\mathrm{K}_{2} \mathrm{HPO}_{4}$ as a base. The reaction protocols demonstrated fast reaction rates (typically within $20 \mathrm{~min}$ ), generally high yields, an absence of noticeable epimerization for stereogenic centers adjacent to carbonyl group, and a simple isolation procedure for solid amide products. In addition to faster rates of solvent-free amide couplings in contrast to the solution-based protocols, the absence of solvent eliminated reagent compatibility issues (e.g. COMU and DMF), greatly reduced the amount of waste generated, and significantly attenuated safety risks. The dual role of $\mathrm{K}_{2} \mathrm{HPO}_{4}$, as both a base and an activating reagent for a carboxylic acid substrate, was also manifested. The rapid formation (within 60-90 min) of amide products was observed for even challenging coupling partners, such as sterically hindered carboxylic acids and poor nucleophilic amines, within the TCFH/ $\mathrm{K}_{2} \mathrm{HPO}_{4}$ and TCFH/NMI reagent systems. However, full amidation of polyfunctionalized substrates, e.g., biotin[6]uril, was found to be especially challenging, even though the single amide bond itself is not difficult to form. Highly reactive coupling conditions (TCFH/NMI), prolonged reaction times (60-90 min), and suitable LAG additives (EtOAc) are essential for producing the hexa-amide 25 in high yield and purity. This efficient and environmentally benign synthetic methodology is useful for preparation of analogues of a new supramolecular host $\mathbf{2 5}$, as well as for synthesis of peptides and amides, which could be used in various fields of applied chemistry.

\section{Notes}

The authors declare no competing financial interest.

\section{ASSOCIATED CONTENT}

Supporting Information: experimental protocols, additional experimental data, copies of NMR spectra, HPLC chromatograms.

\section{AUTHOR INFORMATION}

\section{Corresponding Authors}

* Riina Aav. E-mail: riina.aav@taltech.ee and

* Dzmitry Kananovich. E-mail: dzmitry.kananovich@taltech.ee 


\section{Funding}

The research was supported by the European Union's H2020-FETOPEN grant 828779 (INITIO). Funding from the Estonian Research Council grant PRG399 and support from COST Action CA18112 "Mechanochemistry for Sustainable Industry" and the ERDF CoE in Molecular Cell Engineering 2014- 2020.4.01.15-0013 are gratefully acknowledged.

\section{ACKNOWLEDGMENTS}

We are grateful to Prof. Nicholas Gathergood for infusing us with his passion for green chemistry and the popularization of green metrics during his work at Tallinn University of Technology (2015-2019). Additionally, we acknowledge Dr. Alexander-Mati Müürisepp (TalTech) for measuring IR spectra.

\section{ABBREVIATIONS}

AE, Atom economy; CDI, N,N'-carbonyldiimidazole; COMU, (1-Cyano-2-ethoxy-2oxoethylidenaminooxy)dimethylamino-morpholinocarbenium hexafluorophosphate; DCC, dicyclohexylcarbodiimide; DCM, dichloromethane; DIPEA, $N, N$-diisopropylethylamine; DMAP, 4-dimethylaminopyridine; DMF, N,N-dimethylformamide; $\quad$ EDC, 1-ethyl-3-(3dimethylaminopropyl) carbodiimide; HATU, Hexafluorophosphate Azabenzotriazole Tetramethyl Uronium; HBTU, Hexafluorophosphate Benzotriazole Tetramethyl Uronium; HPLC, High Performance Liquid Chromatography; LAG, Liquid Assisted Grinding; MSDS, Material safety data sheet; NMI, $N$-methylimidazole; Oxyma, ethyl cyanohydroxyiminoacetate; PMI, process

mass intensity; RME, reaction mass efficiency; TCFH, $N, N, N^{\prime}, N^{\prime}$ tetramethylchloroformamidinium hexafluorophosphate; TCT, 2,4,6-trichloro-1,3,5-triazine.

\section{REFERENCES}

(1) Boström, J.; Brown, D. G.; Young, R. J.; Keserü, G. M. Expanding the Medicinal Chemistry Synthetic Toolbox. Nat. Rev. Drug Discov. 2018, 17, 709-727.

(2) Constable, D. J. C.; Dunn, P. J.; Hayler, J. D.; Humphrey, G. R.; Leazer, Jr., J. L.; Linderman, R. J.; Lorenz, K.; Manley, J.; Pearlman, B. A.; Wells, A.; Zaks, A.; Zhang, T. Y. Key Green Chemistry Research Areas-a Perspective from Pharmaceutical Manufacturers. Green Chem. 2007, 9, 411420.

Bryan, M. C.; Dunn, P. J.; Entwistle, D.; Gallou, F.; Koenig, S. G.; Hayler, J. D.; Hickey, M. R.; Hughes, S.; Kopach, M. E.; Moine, G.; Richardson, P.; Roschangar, F.; Steven, A.; Weiberth, F. J. Key Green Chemistry Research Areas from a Pharmaceutical Manufacturers' Perspective 
Revisited. Green Chem. 2018, 20, 5082-5103.

(4) Dalu, F.; Scorciapino, M. A.; Cara, C.; Luridiana, A.; Musinu, A.; Casu, M.; Secci, F.; Cannas, C. A Catalyst-Free, Waste-Less Ethanol-Based Solvothermal Synthesis of Amides. Green Chem. 2018, 20, 375-381.

(5) Charville, H.; Jackson, D. A.; Hodges, G.; Whiting, A.; Wilson, M. R. The Uncatalyzed Direct Amide Formation Reaction - Mechanism Studies and the Key Role of Carboxylic Acid H-Bonding. Eur. J. Org. Chem. 2011, 5981-5990.

(6) Gelens, E.; Smeets, L.; Sliedregt, L. A. J. M.; van Steen, B. J.; Kruse, C. G.; Leurs, R.; Orru, R. V. A. An Atom Efficient and Solvent-Free Synthesis of Structurally Diverse Amides Using Microwaves. Tetrahedron Lett. 2005, 46, 3751-3754.

(7) Dunetz, J. R.; Magano, J.; Weisenburger, G. A. Large-Scale Applications of Amide Coupling Reagents for the Synthesis of Pharmaceuticals. Org. Process Res. Dev. 2016, 20, 140-177.

(8) Valeur, E.; Bradley, M. Amide Bond Formation: Beyond the Myth of Coupling Reagents. Chem. Soc. Rev. 2009, 38, 606-631.

(9) Montalbetti, C. A. G. N.; Falque, V. Amide Bond Formation and Peptide Coupling. Tetrahedron 2005, 61, 10827-10852.

(10) Trobro, S.; Åqvist, J. Mechanism of Peptide Bond Synthesis on the Ribosome. Proc. Natl. Acad. Sci. 2005, 102, 12395-12400.

(11) Goswami, A.; Van Lanen, S. G. Enzymatic Strategies and Biocatalysts for Amide Bond Formation: Tricks of the Trade Outside of the Ribosome. Mol. BioSyst. 2015, 11, 338-353.

(12) Philpott, H. K.; Thomas, P. J.; Tew, D.; Fuerst, D. E.; Lovelock, S. L. A Versatile Biosynthetic Approach to Amide Bond Formation. Green Chem. 2018, 20, 3426-3431.

(13) Petchey, M. R.; Grogan, G. Enzyme-Catalysed Synthesis of Secondary and Tertiary Amides. Adv. Synth. Catal. 2019, 361, 3895-3914.

(14) El-Faham, A.; Albericio, F. Peptide Coupling Reagents, More than a Letter Soup. Chem. Rev. 2011, 111, 6557-6602.

(15) Han, S.-Y.; Kim, Y.-A. Recent Development of Peptide Coupling Reagents in Organic Synthesis. Tetrahedron 2004, 60, 2447-2467.

(16) de Figueiredo, R. M.; Suppo, J.-S.; Campagne, J.-M. Nonclassical Routes for Amide Bond Formation. Chem. Rev. 2016, 116, 12029-12122.

(17) Sabatini, M. T.; Boulton, L. T.; Sneddon, H. F.; Sheppard, T. D. A Green Chemistry Perspective on Catalytic Amide Bond Formation. Nat. Catal. 2019, 2, 10-17.

(18) Pattabiraman, V. R.; Bode, J. W. Rethinking Amide Bond Synthesis. Nature 2011, 480, 471-479.

(19) Massolo, E.; Pirola, M.; Benaglia, M. Amide Bond Formation Strategies: Latest Advances on a Dateless Transformation. Eur. J. Org. Chem. 2020, https://doi.org/10.1002/ejoc.202000080.

(20) Constable, D. J. C.; Jimenez-Gonzalez, C.; Henderson, R. K. Perspective on Solvent Use in the Pharmaceutical Industry. Org. Process Res. Dev. 2007, 11, 133-137.

(21) MacMillan, D. S.; Murray, J.; Sneddon, H. F.; Jamieson, C.; Watson, A. J. B. Evaluation of Alternative Solvents in Common Amide Coupling Reactions: Replacement of Dichloromethane and $N, N$-Dimethylformamide. Green Chem. 2013, 15, 596-600.

(22) Do, J.-L.; Friščić, T. Chemistry 2.0: Developing a New, Solvent-Free System of Chemical Synthesis Based on Mechanochemistry. Synlett 2017, 28, 2066-2092.

(23) James, S. L.; Adams, C. J.; Bolm, C.; Braga, D.; Collier, P.; Friščić, T.; Grepioni, F.; Harris, K. D. M.; Hyett, G.; Jones, W.; Krebs, A.; Mack, J.; Main, L.; Orpen, A. G.; Parkin, I. P.; Shearouse, W. C.; Steed, J. W.; Waddell, D. C. Mechanochemistry: Opportunities for New and Cleaner Synthesis. Chem. Soc. Rev. 2012, 41, 413-447.

(24) Do, J.-L.; Friščić, T. Mechanochemistry: A Force of Synthesis. ACS Cent. Sci. 2017, 3, 13-19.

(25) Margetić, D.; Štrukil, V. Chapter 3 - Carbon-Nitrogen Bond-Formation Reactions; Margetić, D., 
Štrukil, V., Eds.; Elsevier: Boston, 2016; pp 141-233.

(26) Kaupp, G.; Schmeyers, J.; Boy, J. Quantitative Solid-State Reactions of Amines with Carbonyl Compounds and Isothiocyanates. Tetrahedron 2000, 56, 6899-6911.

(27) Cao, Q.; Crawford, D. E.; Shi, C.; James, S. L. Greener Dye Synthesis: Continuous, Solvent-Free Synthesis of Commodity Perylene Diimides by Twin-Screw Extrusion. Angew. Chemie Int. Ed. 2020, 59, 4478-4483.

(28) Declerck, V.; Nun, P.; Martinez, J.; Lamaty, F. Solvent-Free Synthesis of Peptides. Angew. Chemie Int. Ed. 2009, 48, 9318-9321.

(29) Hernández, J. G.; Juaristi, E. Green Synthesis of $\alpha, \beta$ - and $\beta, \beta$-Dipeptides under Solvent-Free Conditions. J. Org. Chem. 2010, 75, 7107-7111.

(30) Bonnamour, J.; Métro, T.-X.; Martinez, J.; Lamaty, F. Environmentally Benign Peptide Synthesis Using Liquid-Assisted Ball-Milling: Application to the Synthesis of Leu-Enkephalin. Green Chem. 2013, 15, 1116-1120.

(31) Gonnet, L.; Tintillier, T.; Venturini, N.; Konnert, L.; Hernandez, J.-F.; Lamaty, F.; Laconde, G.; Martinez, J.; Colacino, E. N-Acyl Benzotriazole Derivatives for the Synthesis of Dipeptides and Tripeptides and Peptide Biotinylation by Mechanochemistry. ACS Sustain. Chem. Eng. 2017, 5, 2936-2941.

(32) Métro, T.-X.; Bonnamour, J.; Reidon, T.; Sarpoulet, J.; Martinez, J.; Lamaty, F. Mechanosynthesis of Amides in the Total Absence of Organic Solvent from Reaction to Product Recovery. Chem. Commun. 2012, 48, 11781-11783.

(33) Duangkamol, C.; Jaita, S.; Wangngae, S.; Phakhodee, W.; Pattarawarapan, M. An Efficient Mechanochemical Synthesis of Amides and Dipeptides Using 2,4,6-Trichloro-1,3,5-Triazine and $\mathrm{PPh}_{3}$. RSC Adv. 2015, 5, 52624-52628.

(34) Hernández, J. G.; Ardila-Fierro, K. J.; Crawford, D.; James, S. L.; Bolm, C. Mechanoenzymatic Peptide and Amide Bond Formation. Green Chem. 2017, 19, 2620-2625.

(35) Ardila-Fierro, K. J.; Crawford, D. E.; Körner, A.; James, S. L.; Bolm, C.; Hernández, J. G. PapainCatalysed Mechanochemical Synthesis of Oligopeptides by Milling and Twin-Screw Extrusion: Application in the Juliá-Colonna Enantioselective Epoxidation. Green Chem. 2018, 20, 1262-1269.

(36) Štrukil, V.; Bartolec, B.; Portada, T.; Đilović, I.; Halasz, I.; Margetić, D. One-Pot Mechanosynthesis of Aromatic Amides and Dipeptides from Carboxylic Acids and Amines. Chem. Commun. 2012, 48, 12100-12102.

(37) Porte, V.; Thioloy, M.; Pigoux, T.; Métro, T.-X.; Martinez, J.; Lamaty, F. Peptide Mechanosynthesis by Direct Coupling of N-Protected $\alpha$-Amino Acids with Amino Esters. Eur. J. Org. Chem. 2016, 3505-3508.

(38) Aav, R.; Shmatova, E.; Reile, I.; Borissova, M.; Topić, F.; Rissanen, K. New Chiral Cyclohexylhemicucurbit[6]Uril. Org. Lett. 2013, 15, 3786-3789.

(39) Prigorchenko, E.; Öeren, M.; Kaabel, S.; Fomitšenko, M.; Reile, I.; Järving, I.; Tamm, T.; Topić, F.; Rissanen, K.; Aav, R. Template-Controlled Synthesis of Chiral Cyclohexylhemicucurbit[8]Uril. Chem. Commun. 2015, 51, 10921-10924.

(40) Kaabel, S.; Adamson, J.; Topić, F.; Kiesilä, A.; Kalenius, E.; Öeren, M.; Reimund, M.; Prigorchenko, E.; Lõokene, A.; Reich, H. J.; et al. Chiral Hemicucurbit[8]Uril as an Anion Receptor: Selectivity to Size, Shape and Charge Distribution. Chem. Sci. 2017, 8, 2184-2190.

(41) Prigorchenko, E.; Kaabel, S.; Narva, T.; Baškir, A.; Fomitšenko, M.; Adamson, J.; Järving, I.; Rissanen, K.; Tamm, T.; Aav, R. Formation and Trapping of the Thermodynamically Unfavoured Inverted-Hemicucurbit[6]Uril. Chem. Commun. 2019, 55, 9307-9310.

(42) Ustrnul, L.; Kaabel, S.; Burankova, T.; Martõnova, J.; Adamson, J.; Konrad, N.; Burk, P.; Borovkov, V.; Aav, R. Supramolecular Chirogenesis in Zinc Porphyrins by Enantiopure Hemicucurbit[n]Urils (n $=6$, 8). Chem. Commun. 2019, 55, 14434-14437. 
(43) Kaabel, S.; Stein, R. S.; Fomitšenko, M.; Järving, I.; Friščić, T.; Aav, R. Size-Control by Anion Templating in Mechanochemical Synthesis of Hemicucurbiturils in the Solid State. Angew. Chemie Int. Ed. 2019, 58, 6230-6234.

(44) Lisbjerg, M.; Jessen, B. M.; Rasmussen, B.; Nielsen, B. E.; Madsen, A. Ø.; Pittelkow, M. Discovery of a Cyclic $6+6$ Hexamer of D-Biotin and Formaldehyde. Chem. Sci. 2014, 5, 2647-2650.

(45) Lisbjerg, M.; Nielsen, B. E.; Milhøj, B. O.; Sauer, S. P. A.; Pittelkow, M. Anion Binding by Biotin[6]Uril in Water. Org. Biomol. Chem. 2015, 13, 369-373.

(46) Lisbjerg, M.; Valkenier, H.; Jessen, B. M.; Al-Kerdi, H.; Davis, A. P.; Pittelkow, M. Biotin[6]Uril Esters: Chloride-Selective Transmembrane Anion Carriers Employing $\mathrm{C}-\mathrm{H} \cdots$ Anion Interactions. J. Am. Chem. Soc. 2015, 137, 4948-4951.

(47) El-Faham, A.; Funosas, R. S.; Prohens, R.; Albericio, F. COMU: A Safer and More Effective Replacement for Benzotriazole-Based Uronium Coupling Reagents. Chem. - A Eur. J. 2009, 15, 9404-9416.

(48) El-Faham, A.; Subirós-Funosas, R.; Albericio, F. A Novel Family of Onium Salts Based Upon Isonitroso Meldrum's Acid Proves Useful as Peptide Coupling Reagents. Eur. J. Org. Chem. 2010, 3641-3649.

(49) El-Faham, A.; Albericio, F. COMU: A Third Generation of Uronium-Type Coupling Reagents. J. Pept. Sci. 2010, 16, 6-9.

(50) Friščić, T.; Childs, S. L.; Rizvi, S. A. A.; Jones, W. The Role of Solvent in Mechanochemical and Sonochemical Cocrystal Formation: A Solubility-Based Approach for Predicting Cocrystallisation Outcome. CrystEngComm 2009, 11, 418-426.

(51) Prat, D.; Hayler, J.; Wells, A. A Survey of Solvent Selection Guides. Green Chem. 2014, 16, 45464551.

(52) Prat, D.; Wells, A.; Hayler, J.; Sneddon, H.; McElroy, C. R.; Abou-Shehada, S.; Dunn, P. J. CHEM21 Selection Guide of Classical- and Less Classical-Solvents. Green Chem. 2016, 18, 288-296.

(53) Handbook of Biochemistry and Molecular Biology; Lundblad, R. L., Macdonald, F., Eds.; CRC Press: Boca Raton, 2010.

(54) Sperry, J. B.; Minteer, C. J.; Tao, J.; Johnson, R.; Duzguner, R.; Hawksworth, M.; Oke, S.; Richardson, P. F.; Barnhart, R.; Bill, D. R.; Giusto, R. A.; Weaver III, J. D. Thermal Stability Assessment of Peptide Coupling Reagents Commonly Used in Pharmaceutical Manufacturing. Org. Process Res. Dev. 2018, 22, 1262-1275.

(55) McKnelly, K. J.; Sokol, W.; Nowick, J. S. Anaphylaxis Induced by Peptide Coupling Agents: Lessons Learned from Repeated Exposure to HATU, HBTU, and HCTU. J. Org. Chem. 2020, 85, 1764-1768.

(56) Isidro-Llobet, A.; Kenworthy, M. N.; Mukherjee, S.; Kopach, M. E.; Wegner, K.; Gallou, F.; Smith, A. G.; Roschangar, F. Sustainability Challenges in Peptide Synthesis and Purification: From R\&D to Production. J. Org. Chem. 2019, 84, 4615-4628.

(57) Subirós-Funosas, R.; Nieto-Rodriguez, L.; Jensen, K. J.; Albericio, F. COMU: Scope and Limitations of the Latest Innovation in Peptide Acyl Transfer Reagents. J. Pept. Sci. 2013, 19, 408-414.

(58) Kumar, A.; Jad, Y. E.; de la Torre, B. G.; El-Faham, A.; Albericio, F. Re-Evaluating the Stability of COMU in Different Solvents. J. Pept. Sci. 2017, 23, 763-768.

(59) McElroy, C. R.; Constantinou, A.; Jones, L. C.; Summerton, L.; Clark, J. H. Towards a Holistic Approach to Metrics for the 21st Century Pharmaceutical Industry. Green Chem. 2015, 17, 31113121.

(60) MSDS data was retrieved from on-line catalogues of chemicals, e.g. https://www.sigmaaldrich.com/ or http://www.fluorochem.co.uk/.

(61) Beutner, G. L.; Young, I. S.; Davies, M. L.; Hickey, M. R.; Park, H.; Stevens, J. M.; Ye, Q. TCFH-NMI: Direct Access to N-Acyl Imidazoliums for Challenging Amide Bond Formations. Org. Lett. 2018, 20, 4218-4222. 
(62) Lopez, F. J.; Ferriño, S. A.; Reyes, M. S.; Román, R. Asymmetric Transformation of the Second Kind of Racemic Naproxen. Tetrahedron: Asymmetry 1997, 8, 2497-2500.

(63) Blasco, M. A.; Gröger, H. Organocatalytic Racemization of $\alpha$-Aryl Propionates in the Presence of Water. Synth. Commun. 2013, 43, 9-15.

(64) Chen, C.-S.; Chen, T.; Shieh, W.-R. Metabolic Stereoisomeric Inversion of 2-Arylpropionic Acids. On the Mechanism of Ibuprofen Epimerization in Rats. Biochim. Biophys. Acta-Gen. Subj. 1990, 1033, 1-6.

(65) Gao, X.; Deng, H.; Tang, G.; Liu, Y.; Xu, P.; Zhao, Y. Intermolecular Phosphoryl Transfer of NPhosphoryl Amino Acids. Eur. J. Org. Chem. 2011, 3220-3228.

(66) Di Sabato, G.; Jencks, W. P. Mechanism and Catalysis of Reactions of Acyl Phosphates. I. Nucleophilic Reactions. J. Am. Chem. Soc. 1961, 83, 4393-4400.

(67) Kluger, R.; Huang, Z. Acyl Pyrophosphates: Activated Analogs of Pyrophosphate Monoesters Permitting New Designs for Inactivation of Targeted Enzymes. J. Am. Chem. Soc. 1991, 113, 51245125.

(68) Biron, J.-P.; Pascal, R. Amino Acid N-Carboxyanhydrides: Activated Peptide Monomers Behaving as Phosphate-Activating Agents in Aqueous Solution. J. Am. Chem. Soc. 2004, 126, 9198-9199.

(69) Leman, L. J.; Orgel, L. E.; Ghadiri, M. R. Amino Acid Dependent Formation of Phosphate Anhydrides in Water Mediated by Carbonyl Sulfide. J. Am. Chem. Soc. 2006, 128, 20-21.

(70) Twibanire, J. K.; Grindley, T. B. Efficient and Controllably Selective Preparation of Esters Using Uronium-Based Coupling Agents. Org. Lett. 2011, 13, 2988-2991.

(71) Xiong, B.; Hu, C.; Gu, J.; Yang, C.; Zhang, P.; Liu, Y.; Tang, K. Efficient and Controllable Esterification of $\mathrm{P}(\mathrm{O})-\mathrm{OH}$ Compounds Using Uronium-Based Salts. ChemistrySelect 2017, 2, 3376-3380.

(72) Larrivée-Aboussafy, C.; Jones, B. P.; Price, K. E.; Hardink, M. A.; McLaughlin, R. W.; Lillie, B. M.; Hawkins, J. M.; Vaidyanathan, R. DBU Catalysis of $N, N^{\prime}$-Carbonyldiimidazole-Mediated Amidations. Org. Lett. 2010, 12, 324-327.

(73) Yawer, M. A.; Havel, V.; Sindelar, V. A Bambusuril Macrocycle That Binds Anions in Water with High Affinity and Selectivity. Angew. Chemie Int. Ed. 2015, 54, 276-279.

(74) Lizal, T.; Sindelar, V. Bambusuril Anion Receptors. Isr. J. Chem. 2018, 58, 326-333.

(75) Andersen, N. N.; Lisbjerg, M.; Eriksen, K.; Pittelkow, M. Hemicucurbit[n]Urils and Their Derivatives - Synthesis and Applications. Isr. J. Chem. 2018, 58, 435-448.

(76) Masson, E.; Ling, X.; Joseph, R.; Kyeremeh-Mensah, L.; Lu, X. Cucurbituril Chemistry: A Tale of Supramolecular Success. RSC Adv. 2012, 2, 1213-1247.

(77) Aav, R.; Kaabel, S.; Fomitšenko, M. Cucurbiturils: Synthesis, Structures, Formation Mechanisms, and Nomenclature; In Comprehensive Supramolecular Chemistry II Atwood, J. L., Ed.; Elsevier: Oxford, 2017; pp 203-220

(78) Hachmann, J.; Lebl, M. Search for Optimal Coupling Reagent in Multiple Peptide Synthesizer. Pept. Sci. 2006, 84, 340-347. 